\title{
BODY COMPOSITION IN CHILDREN AND YOUNG PATIENTS AFFECTED BY CHRONIC DISEASES
}

S.Vai ${ }^{1}$, ML Bianchi ${ }^{1}$, C Colombo ${ }^{2}$, L. Ghio ${ }^{2}$, F. Corona ${ }^{2}$, G. Baranello ${ }^{3}$

${ }^{1}$ Experimental Laboratory for Children's Bone Metabolism Research, Bone Metabolism Unit, Istituto Auxologico Italiano IRCCS;

${ }^{2}$ Clinica Pediatrica, Fondazione IRCCS Ca' Granda Ospedale Maggiore Policlinico; ${ }^{3}$ Istituto Neurologico C. Besta, IRCCS; Milano Italy.

ADDRESS FOR CORRESPONDENCE: s.vai@auxologico.it

\section{PATIENTS \& METHODS}

We analyzed body composition (DXA, Hologic) in 334 young patients (aged 3-24 years) with chronic diseases, most of them on longterm glucocorticoid (GC) treatment, and monitored its changes over 3 to 14 years (6.9 6.2 years).

Bone Mineral Content (BMC), Fat Mass (FM) and Fat-Free Mass (FFM) were measured on Total Body Less Head (TBLH), trunk, upper limbs, lower limbs. Regional values of BMC, FM, FFM were expressed as percentages of total and compared with age- and sexmatched healthy Italian controls. Body Mass Index (BMI) was also measured.

\section{RESULTS}

In 159 patients with nephrotic syndrome, connective tissue diseases, asthma, autoimmune hepatitis, or transplants, GCs had major effects on trunk BMC and FM, related to cumulative dose. Independently of disease and age, BMC decrease and FM increase were higher during the first year of treatment, and continued more slowly thereafter.

\section{9 children/adolescents (3-20 yrs)}
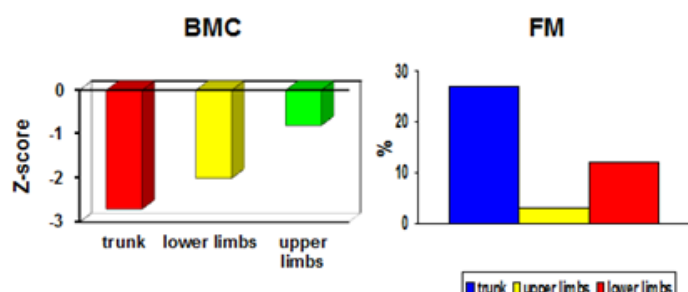

Increase of trunk fat
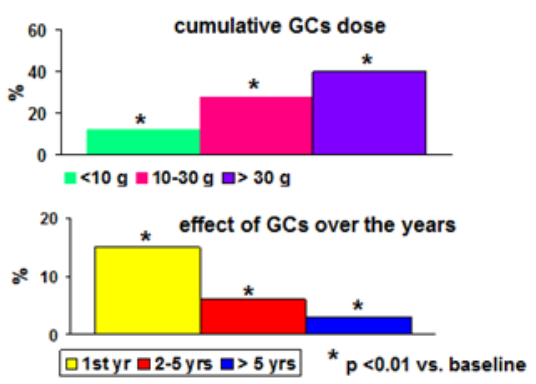

Reduction of trunk BMC
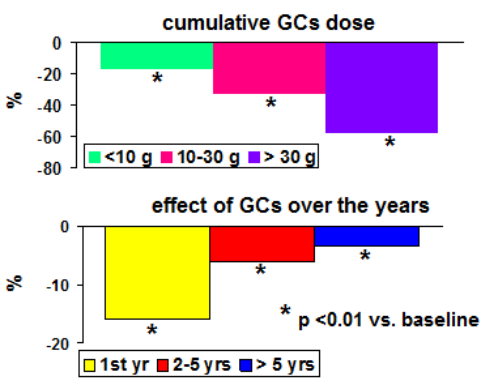

In 39 walking boys with Duchenne Muscular Dystrophy (DMD), BMC was low for age, and more reduced at lower than upper limbs. At lower limbs, FM progressively increased and FFM decreased over time. The FFM decrease correlated with changes in muscle strength (evaluated by Manual Muscle Testing, MRC scale).

39 boys with DMD (4-15 yrs): body composition changes

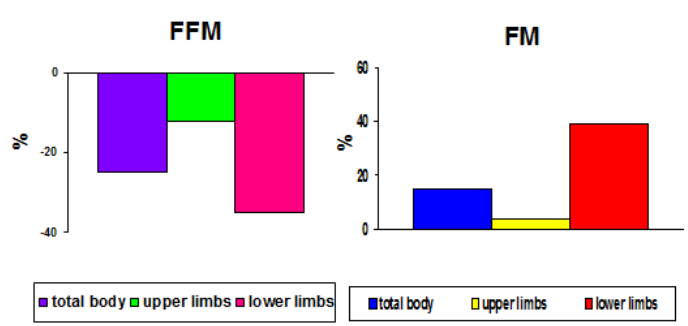

Decrease of lower-limb FFM vs. decrease of muscle strength

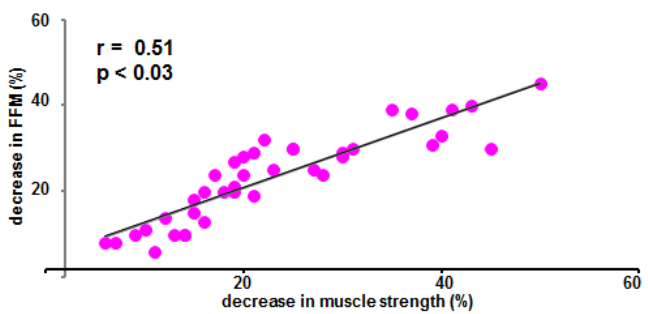

In 136 patients affected by Cystic Fibrosis (CF), body composition analysis showed decreased FFM and FM with respect to controls in both sexes, for both TB and the 3 sub-regions. We observed significant correlations of BMI, FM, FFM with trunk BMC (FM showing the highest correlation); in addition, of BMI with FFM; of FFM with both TB BMC and lower-limbs BMC, as well as with FEV1, a pulmonary function index.

136 CF patients (3-24 yrs): body composition changes FFM

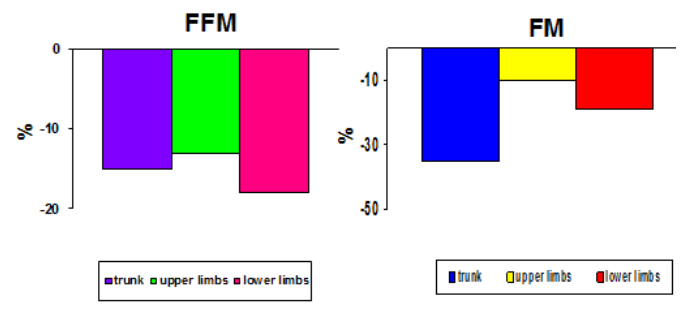

BMC in patients with CF

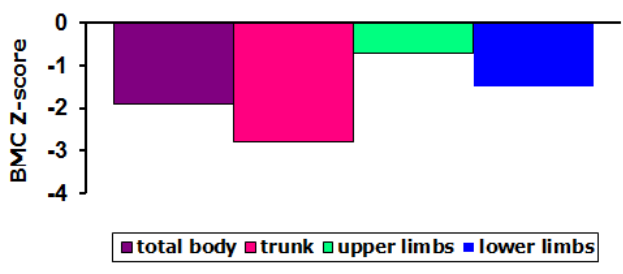

Correlations

\begin{tabular}{|l|c|c|c|r|c|}
\hline & FFM & TB-BMC & $\begin{array}{c}\text { Lower-limb } \\
\text { BMC }\end{array}$ & $\begin{array}{r}\text { Trunk } \\
\text { BMC }\end{array}$ & FEV 1 \\
\hline BMI & $\begin{array}{c}0.62 \\
<0.01\end{array}$ & 0.18 & 0.23 & $\begin{array}{r}0.43 \\
<0.05\end{array}$ & 0.29 \\
\hline FM & - & 0.14 & 0.1 & 0.61 & 0.17 \\
& & & & $<0.01$ & \\
\hline FFM & - & 0.64 & 0.56 & 0.49 & 0.61 \\
& & $<0.01$ & $<0.02$ & $<0.05$ & $<0.01$ \\
\hline FEV $_{1}$ & 0.61 & 0.09 & 0.18 & 0.50 & - \\
& $<0.01$ & & & $<0.03$ & \\
\hline
\end{tabular}

\section{CONCLUSIONS}

In children and young patients, body composition analysis is a powerful tool to evaluate the disease-related deviations from normal, to demonstrate the GC influence on BMC, FM, and FFM, to highlight the relationship between muscular strength/activity and bone. 